\title{
Evaluación de Impacto Ambiental producido por el Uso de Leña en Zonas Rurales de la Región Tacna
}

\section{Environmental Impact Assessment produced by the use of Firewood in Rural Areas of the Tacna Region}

' Hugo Alfredo Torres Muro

\begin{abstract}
RESUMEN:
El $70 \%$ de la población de las zonas rurales, interandinas y altoandinas de muestro país usa biomasa para calefacción. cocción y otras lahores artesanales y costumbristas. En consecuencia. es necesario disponer de datos detallados sobre extracción, consumo de hiomasa y los posibles impactos que pueden estar provocando, para planificar su producción y utilización sostenibles. Este trabajo es producto de varios años de investigación; las condiciones geográficas, climáticas y culturales de la zona en estudio impide realizar una estadia prolongada para la recolección de información de fuentes primarias, sin embargo. con la estimación del consumo de leña y la valoración cuantitativa y cualitativa de impactos ambientales en esta zona, se contribuye a llenar un vacio de información. Los resultados obtenidos nos permiten concluir que el uso de leña en el área de estudio. está provocando impactos ambientales negativos de consecuencias locales y globales. Las autoridades regionales y locales deben planificar en el más corto plazo un programa con tecnologias limpias y renovables para solucionar este problema.
\end{abstract}

Palabras clave: combustión de leña, cocinas tradicionales, impactos ambientales, valoración de impactos.

\section{ABSTRACT:}

The $70 \%$ of population in rural areas, inter-andean and highlands of our country use biomass for heating, cooking and other handmade and of manners works. In consequence, it is necessary to have detailed data on extraction. consumption of biomass and the possible impacts that can be causing, to plan their production and sustainable use. This work is a product of several years of investigation; the geographical, climatic and cultural conditions of the area in study do not let to stay for a long time to carry out information of primary sources, however, with the estimation of the firewood consumption and the quantitative and qualitative valuation of environmental impacts in this area, it is contributed to fill a hole of information. The obtained results allow us to conclude that the firewood use in the study area, is causing negatives environmental impacts of local and global consequences. The regional and local authorities should plan a program with clean and renewable technologies to solve this problem in the shortest term.

Keywords: combustion of wood, traditional kitchens, environmental impacts, assessment of impacts.

\footnotetext{
'Master of Sciencie con mención en Gestión Ambiental y Desarrollo Sostenible. Licenciado en Física. Centro de Energias Renovables de Tacna
} Facultad de Ciencias. Universidad Nacional Jorge Basadre Grohmann 


\section{INTRODUCCIÓN}

En el Perú. en las zonas rurales, el uso de la leña como fuente primaria de energia representa una fracción muy importante de todas las necesidades energéticas de la población: y su escasez es cubierta en muchos casos por el uso de la bosta y la yareta. La población de las zonas rurales costeras, interandinas y altoandinas de la Region Tacna, en su gran mayoría usa biomasa (leña, bosta, yareta) como fuente primaria de energia, en aplicaciones domésticas y artesanales como la preparación de sus alimentos, el teñido de lana y la producción de carbón vegetal. Sus fuentes de extracción son los bosques ubicados lejos de las viviendas y en otros cercanos a sus campos agrícolas o pastizales. Además, en algunos lugares existe un mercado comercial, aunque informal. de leña, que constituye una fuente importante de ingresos para familias rurales

.Las cocinas o estufas usadas para la cocción son tradicionales, estas pueden ser fijas o portátiles $\mathrm{y}$, a veces, tienen una chimenea. Algunas familias hacen su propia estufa de materiales locales: otras buscan el servicio de un artesano, o la compran en las ferias comerciales. Generalmente, estas son simples y de baja eficiencia, pues presentan pérdidas normales entre $80 \%$ y $95 \%$ de energia. Aunque los usuarios tratan de mejorar las estufas, por lo general carecen de los recursos financieros y técnicos para hacerlo adecuadamente. Estas prácticas cotidianas que tienen por tinalidad satisfacer necesidades vitales de la población, y que son generadoras de emisiones de gases de efecto invernadero, pueden estar causando algunos impactos que son necesarios valorarlos y cuantificarlos para determinar si, a pesar de la dispersión de las fuentes puntuales, son lo suficientemente considerables para provocar cambios adversos en el ambiente desde el punto de vista local y global.

La población Rural que involucra este estudio de investigación comprende 1006 habitantes ubicados en el Área de Conservación Regional Vilacota-Maure (ACRVM), distribuidos en los distritos de Tacna, Tarata. Candarave y Palca, según estadisticas del INEI para el año 2005 y estudios realizados por el Gobierno Regional de Tacna en vias de implementar el Plan de Ordenamiento Territorial y el establecimiento de una Zonificación Ecológica Económica.

\section{MATERIALY MÉTODOS}

Con la finalidad de obtener información sobre las formas de recolección, cantidades y usos de leña, tola, bosta y yareta, se realizaron viajes periódicos, principalmente en los meses en que el clima es bueno para viajar y desplazarse a cada uno de los anexos listados al azahar según el mapa del ACRVM. De esta manera se realizaron viajes de estudio en los meses de Agosto y Noviembre del 2007, Agosto y Diciembre del 2008, Setiembre y Diciembre del 2009. En estos viajes se recolectaron datos primarios obtenidos directamente de los habitantes que conforman la muestra de estudio, es una información de primera mano y específica para nuestro estudio.
Para recolectar información relacionados con las variables de estudio. especificamente sobre las formas de recolección, cantidades y usos de leña, tola, bosta y yareta, asi como las costumbres para cocinar, se aplicaron encuestas tipo cuestionarios elaborados por los autores.

Para establecer la linea de base, determinar los impactos sobre la salud de las personas por exposición al $\mathrm{CO}_{2}$ en interiores y estimar la tasa de deforestación de los bosques se recurrió a datos secundarios, provenientes de estudios ya existentes. que fueron elaborados con una finalidad distinta a los objetivos del presente estudio. Estas bases fueron muy útiles en nuestro caso, por no contar con equipos o instrumentos para medir directamente la cantidad de $\mathrm{CO}_{2}$ a la que está expuesta la población objetivo durante la actividad de cocinar sus alimentos. Tampoco contábamos con imágenes satelitales pero si de un modelo matemático, para determinar la tasa de deforestación. En consecuencia usamos la estrategia de establecer una o dos preguntas, para luego buscar las respuestas en la base de datos. elaborada por los autores. Para determinar los impactos ambientales se aplicó el método de redes para la evaluación cualitativa y de matrices valorativas para la evaluación cuantitatival.

\section{RESULTADOSY DISCUSIÓN}

El ACRVM. se considera como un área de uso directo, en ella que se permite el aprovechamiento o extracción de recursos, para aquellos. definidos por el plan de manejo del área. Otros usos deberán ser compatibles con sus objetivos. Se permiten los usos científicos y turisticos. Las modificaciones de actividades y prácticas tradicionales, así como al uso de recursos naturales no renovables, requieren autorización especifica del Gobiemo Regional de Tacna y un monitoreo permanente.

La planificación en el ámbito del ACRVM, como área natural protegida, se realizó el mes de Abril del 2006. Los procesos de planificación se dieron inicialmente en los anexos de Vilacota y Mamaraya, a través de talleres participativos de primer nivel para recopilación de información de línea base en cada localidad.

En el 2007, entre los meses de Julio a Diciembre, la consultora CAMDESO SRL es la encargada de llevar a cabo el proceso de elaboración del Plan Maestro del ACRVM, cuyo informe es el resultado de un proceso participativo, de recopilación. levantamiento y sistematización de información y análisis de variables. Algunos resultados presentados en este Plan, han sido tomados como datos para elaborar la línea de base para el estudio realizado en el presente trabajo, con el debido permiso de la consultora mencionada.

\subsection{Estudio de Línea de base.}

En el proceso de planificación se ha identificado como uno de los principales problemas del área, la inadecuada ordenación del medio natural para mantener la relación hombre naturaleza, la biodiversidad, la prevención de 
riesgos ambientales y la sostenibilidad de los recursos naturales. Existen problemas de pérdida de suelo, que tienen que ver con el inadecuado uso del mismo. La eliminación de la cobertura vegetal debido a un uso inapropiado de los recursos naturales (sobre pastoreo, tala y extracción ilegal de árboles y arbustos, quema de pastos naturales, etc.) deja sin protección el suelo frente al fuerte impacto de precipitaciones torrenciales, aumentando la escorrentía superficial en las pendientes. El diagnóstico de la zona, ha puesto de manifiesto la existencia de una actividad ganadera extensiva y no controlada, la explotación irracional de especies forestales, además de la caza furtiva.

El ACRVM, se encuentra ubicada en los Andes Meridionales del Perú, conformando parte de la cadena occidental de la Cordillera de los Andes, en la zona altoandina de la región Tacna. Administrativamente abarca territorios de los distritos de Palca, en la provincia de Tacna; Susapaya, Ticaco y Tarata, en la provincia de Tarata; y Candarave, en la provincia del mismo nombre. Tiene una extensión superficial de 124 922.97 ha, representando el $7.77 \%$ de la superficie regional. En la Figura 1 el mapa de ubicación de la zona estudiada. El área propuesta contiene una muéstra representativa de ecosistemas y paisajes altoandinos que son un fuerte respaldo y sirven de cobertura a todo el sistema natural, así como el antropogénico. De acuerdo al Sistema de Clasificación de las formaciones vegetales del mundo de Holdridge, aplicado al Perú por Joseph A. Tosi (INRENA 1995), en el ámbito del ACRVM están
Tosi (INRENA 1995), en el ámbito del ACRVM están comprendidas 05 zonas de vida: Matorral desértico Subalpino Templado cálido (md SaTc), Páramo húmedo Subalpino Subtropical (ph SaS), Tundra húmeda Alpino Templado cálido (th ATc), Tundra muy húmeda Alpino Subtropical ( tmh AS), Nival - Subtropical (NS), Nival Templado cálido (Ntc). El ámbito del ACRVM, por encontrarse sobre los 3800 m.s.n.m., ha determinado la práctica de actividades económicas principalmente ganadera (camélidos sudamericanos: alpacas y llamas), sumada a una débil actividad comercial informal. La mayor parte de la población realiza estas actividades solo con fines de subsistencia. Presenta actividades y procesos productivos desarticulados, básicamente orientados a la producción primaria sin mayor grado de procesamiento. Esto entorpece el crecimiento sostenido y el estancamiento relativo de su aparato productivo.

En la Tabla 1, se presentan los valores totales de la cantidad de biomasa vegetal (leña, tola, bosta y yareta), que consumen todas las familias encuestadas, valores calculados para cada semana, cada mes y cada año. De igual manera se consideran los valores promedios de consumo de leña por un lado y tola, bosta y yareta en kilogramos por semana, por mes y por año que consume cada familia encuestada. Finalmente se presentan los resultados de los valores promedios de consumo biomasa vegetal (leña, tola, bosta y yareta) en kilogramos por semana, por mes y por año que consume cada familia.

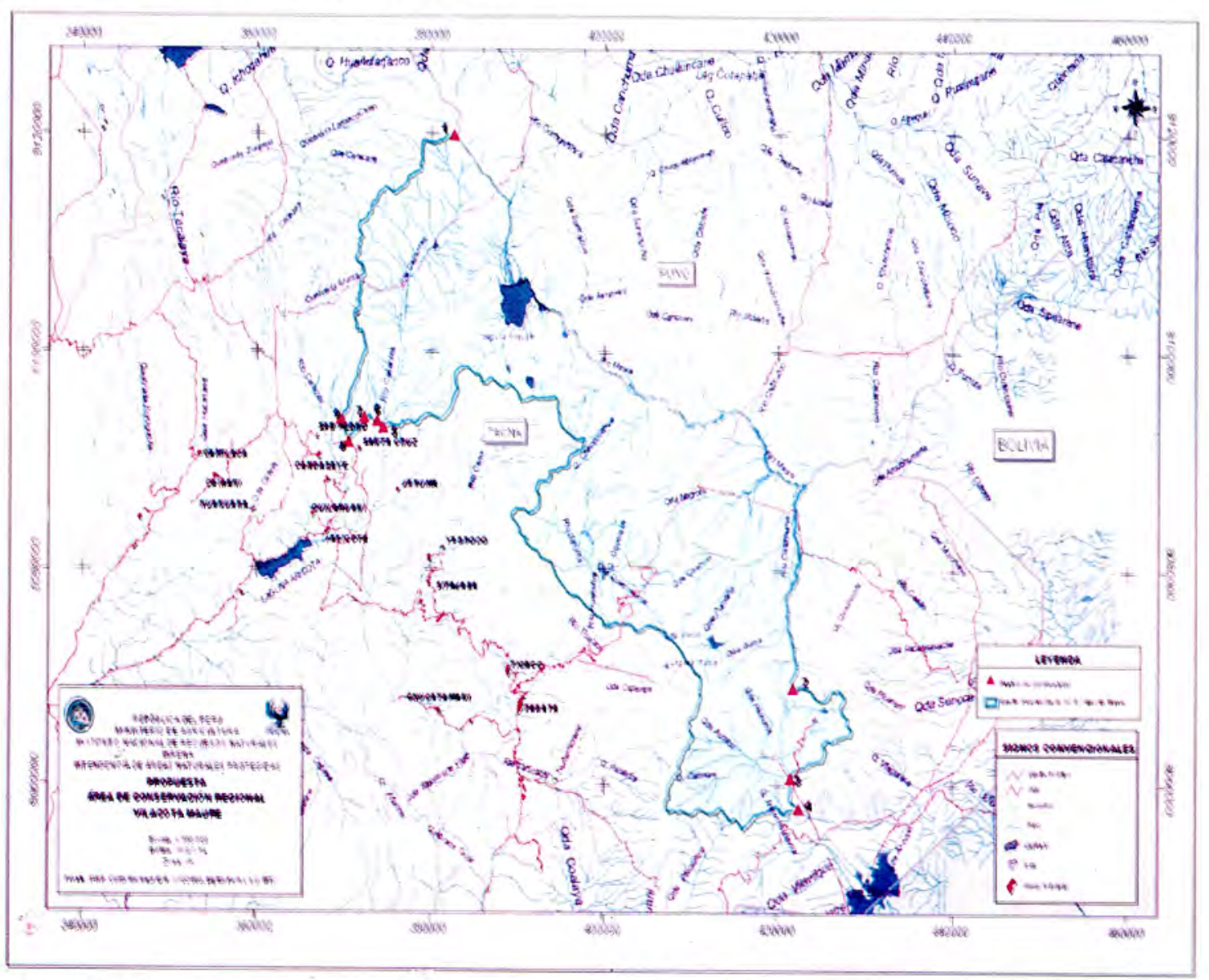

Figura 1. Mapa de ubicación del Área de Conservación Regional Vilacota-Maure de la Región Tacna Fuente: Plan Maestro del ACRVM. CAMDESO SRL.- GRT (2007) 
3.2 Estimación de la cantidad de $\mathrm{CO}_{2}$ emitida a la atmósfera.

Teniendo en cuenta a Dieter Seifert y D-Neuoetting (2000), Dieter Seifert (1998), Antonio Creus S. (2004) la combustión de un kilogramo de leña emite $1.83 \mathrm{~kg}$ de $\mathrm{CO}_{2}$; esto se debe a que en la leña la mitad de su masa es carbón (C) y si la relación entre el peso molecular del $\mathrm{CO}_{2}$ y el C es de $44 / 12$ entonces; $1 \mathrm{~kg}$ leña produce: 0.5 $\left(44 / 12 \mathrm{~kg}\right.$ de $\left.\mathrm{CO}_{2}\right)=1.83 \mathrm{~kg}$ de $\mathrm{CO}_{2}$. La masa total MT de $\mathrm{CO}_{2}$ que emiten las 255 familias que ocupan el ACRVM será:

$$
\mathrm{M}_{\mathrm{TCO} 2}=5,7 \times 255=1453,5 \frac{\mathrm{T}}{\mathrm{a}}
$$

\subsection{Identificación de Impactos Ambientales (IIA).}

Para la identificación de los componentes ambientales e impactos ambientales se utilizará las matrices de causa y efecto.
Componentes Ambientales. En la Figura 2, se muestra el resultado de la identificación de componentes ambientales como el aire, suelo, flora, fauna, social, económico y de interés humano, susceptibles de ser impactados por las actividades impactantes como: extracción de leña, búsqueda, recolección y traslado de leña, cocción de alimentos usando leña (queñoa, tola, yareta, bosta) en cocinas tradicionales y exposición a gases emitidos en el proceso de combustión en cocinas tradicionales.

Impactos Ambientales. En la Figura 3 se muestra, el resultado de la identificación de impactos ambientales sobre el medio abiótico aire, suelo, medio biótico, flora, fauna y el medio social, económico y de interés humano, susceptibles de ser impactados por las actividades impactantes como: extracción de leña, búsqueda, recolección y traslado de leña, cocción de alimentos usando leña en cocinas tradicionales y exposición a gases emitidos en el proceso de combustión en cocinas tradicionales.

Tabla $\mathrm{N}^{\circ}$ 1: Valores Promedios de Uso de Leña, Yareta y Bosta en el Acrvm - Región Tacna

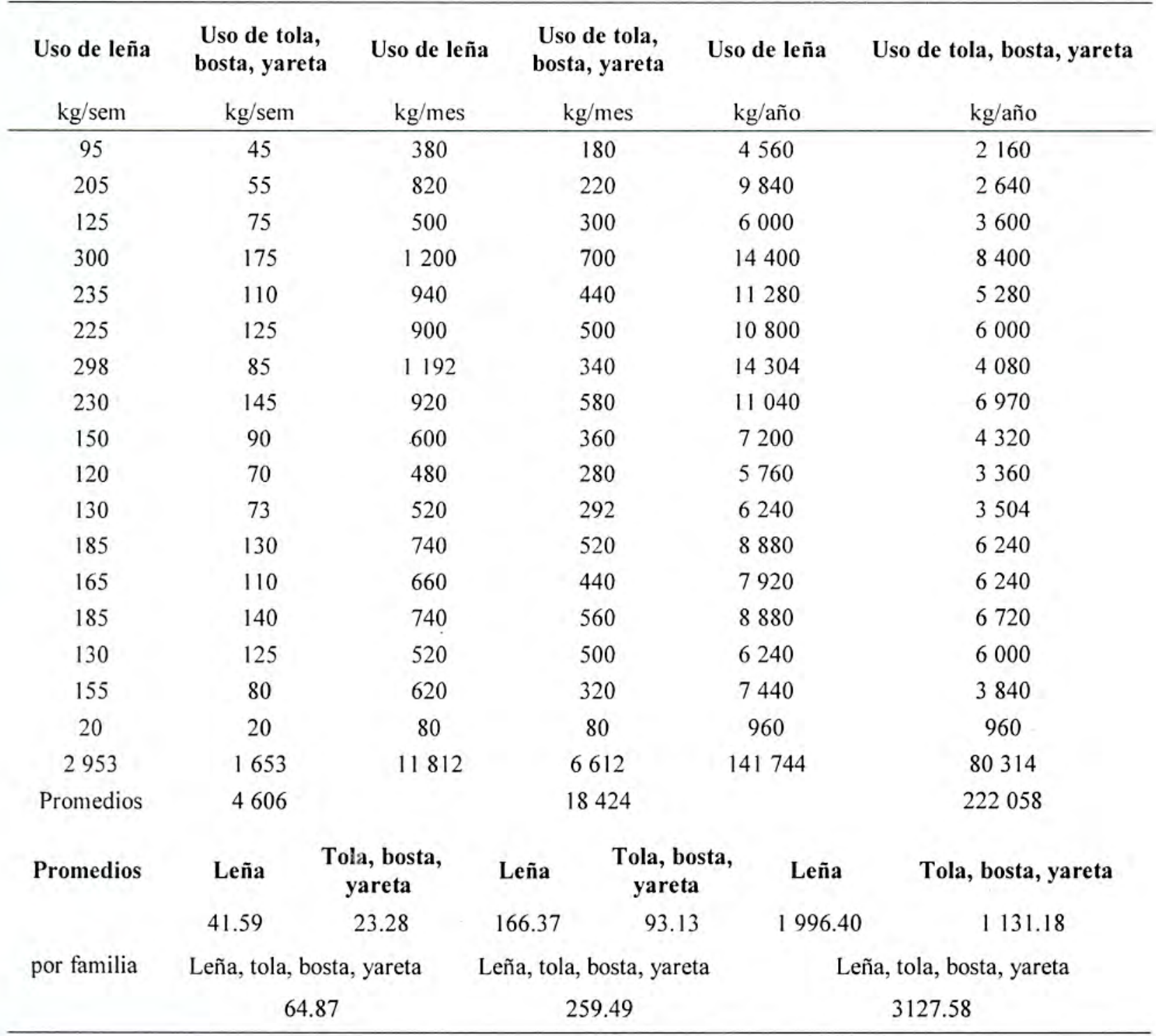




\subsection{Evaluación de Impactos Ambientales (EIA).}

La metodología utilizada para la evaluación de los impactos ambientales, se logra con el análisis de los aspectos ambientales identificados y su interacción con los componentes ambientales que podrian verse afectados.

\subsection{Evaluación Cualitativa.}

La escala de evaluación cualitativa de los impactos ambientales, se refiere al grado de incidencia de la actividad sobre un determinado componente ambiental. La variación de la calidad ambiental. establece si el cambio producido por las actividades en relación al

\begin{tabular}{|c|c|c|c|c|c|c|c|c|c|c|c|c|c|}
\hline \multirow[b]{3}{*}{ ACTIVIDAD } & \multicolumn{6}{|c|}{ MEdIO FísıCo } & \multicolumn{2}{|c|}{$\begin{array}{l}\text { MEDIO } \\
\text { BIOLÓGICO }\end{array}$} & \multicolumn{5}{|c|}{$\begin{array}{l}\text { MEDIO SOCIECONOMICO Y } \\
\text { CULTURAL }\end{array}$} \\
\hline & \multicolumn{4}{|c|}{ Aire } & \multicolumn{2}{|c|}{ Suelo } & \multirow{2}{*}{ 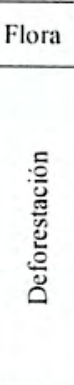 } & \multirow[b]{2}{*}{ 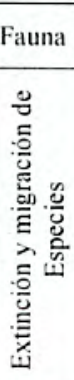 } & \multirow[b]{2}{*}{ 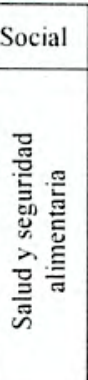 } & \multicolumn{2}{|c|}{ Económico } & \multicolumn{2}{|c|}{$\begin{array}{c}\text { Interés } \\
\text { Humano }\end{array}$} \\
\hline & 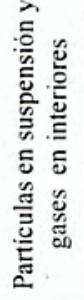 & 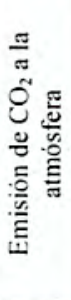 & 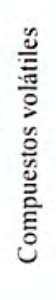 & 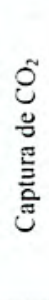 & 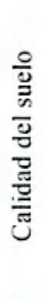 & 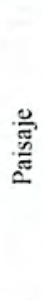 & & & & 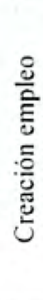 & 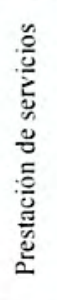 & 语 & 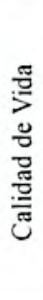 \\
\hline Extracción de Leña & & & & $\mathrm{x}$ & $\mathrm{x}$ & $x$ & $\mathrm{x}$ & $x$ & & $\mathrm{x}$ & $\mathrm{x}$ & $x$ & $x$ \\
\hline $\begin{array}{l}\text { Uso de leña en } \\
\text { Cocción de } \\
\text { alimentos en } \\
\text { cocinas } \\
\text { tradicionales }\end{array}$ & $\mathrm{x}$ & $\mathrm{X}$ & $\mathrm{X}$ & $\mathrm{x}$ & $\mathrm{x}$ & $\mathrm{x}$ & $\mathrm{x}$ & $\mathrm{x}$ & $\mathrm{x}$ & $\mathrm{x}$ & $\mathrm{x}$ & $\mathrm{x}$ & $\mathrm{x}$ \\
\hline $\begin{array}{l}\text { Búsqueda, } \\
\text { recolección y } \\
\text { traslado de leña }\end{array}$ & & & & & & $\mathrm{x}$ & $\mathrm{x}$ & $\mathrm{x}$ & & $\mathrm{x}$ & $\mathrm{x}$ & & $\mathrm{x}$ \\
\hline $\begin{array}{l}\text { Exposición a gases } \\
\text { emitidos en el } \\
\text { proceso de } \\
\text { combustión en } \\
\text { cocinas } \\
\text { tradicionales }\end{array}$ & & & & & & & & & $\mathrm{X}$ & $\mathrm{x}$ & $\mathrm{x}$ & $\mathrm{x}$ & $\mathrm{x}$ \\
\hline
\end{tabular}

Figura 2. Identificación de Componentes Ambientales susceptibles de ser impactantes

\begin{tabular}{|c|c|c|c|}
\hline Componentes Impactantes & $\begin{array}{l}\text { Categoria } \\
\text { Ambiental }\end{array}$ & $\begin{array}{c}\text { Componente } \\
\text { Ambiental }\end{array}$ & $\begin{array}{c}\text { Aspecto } \\
\text { Ambiental }\end{array}$ \\
\hline \multirow{6}{*}{$\begin{array}{l}\text { 1. Extracción de Leña } \\
\text { 2. Búsqueda, recolección y } \\
\text { traslado de leña }\end{array}$} & \multirow{6}{*}{ Medio Físico } & \multirow{4}{*}{ Aire } & Particulas en suspensión y gases de combustión en interiores \\
\hline & & & Compuestos volátiles \\
\hline & & & Captura de $\mathrm{CO}_{2}$ \\
\hline & & & Emisiones de $\mathrm{CO}_{2}$ a la atmósfera \\
\hline & & & Paisaje \\
\hline & & Suelo & Calidad del suelo \\
\hline \multirow{2}{*}{$\begin{array}{l}\text { 3. Uso de leña para } \\
\text { Cocción de alimentos en } \\
\text { cocinas tradicionales }\end{array}$} & \multirow{2}{*}{$\begin{array}{c}\text { Medio } \\
\text { Biológico }\end{array}$} & Flora & Deforestación \\
\hline & & Fauna & Extinción y Migración de especies \\
\hline \multirow{6}{*}{$\begin{array}{l}\text { 4. Exposición a gases } \\
\text { emitidos en el proceso } \\
\text { de combustión en } \\
\text { cocinas tradicionales }\end{array}$} & \multirow{6}{*}{$\begin{array}{c}\text { Socioeconómico } \\
\text { y cultural }\end{array}$} & \multirow{2}{*}{ Social } & Salud y seguridad alimentaria \\
\hline & & & Conflictos socio ambientales \\
\hline & & \multirow{2}{*}{ Económico } & Creación empleo \\
\hline & & & Prestación de servicios \\
\hline & & \multirow{2}{*}{$\begin{array}{l}\text { Interés } \\
\text { humano }\end{array}$} & Migración \\
\hline & & & Alteración del paisaje \\
\hline
\end{tabular}

Figura 3. Matriz de Impactos Potenciales por Componente Ambiental 


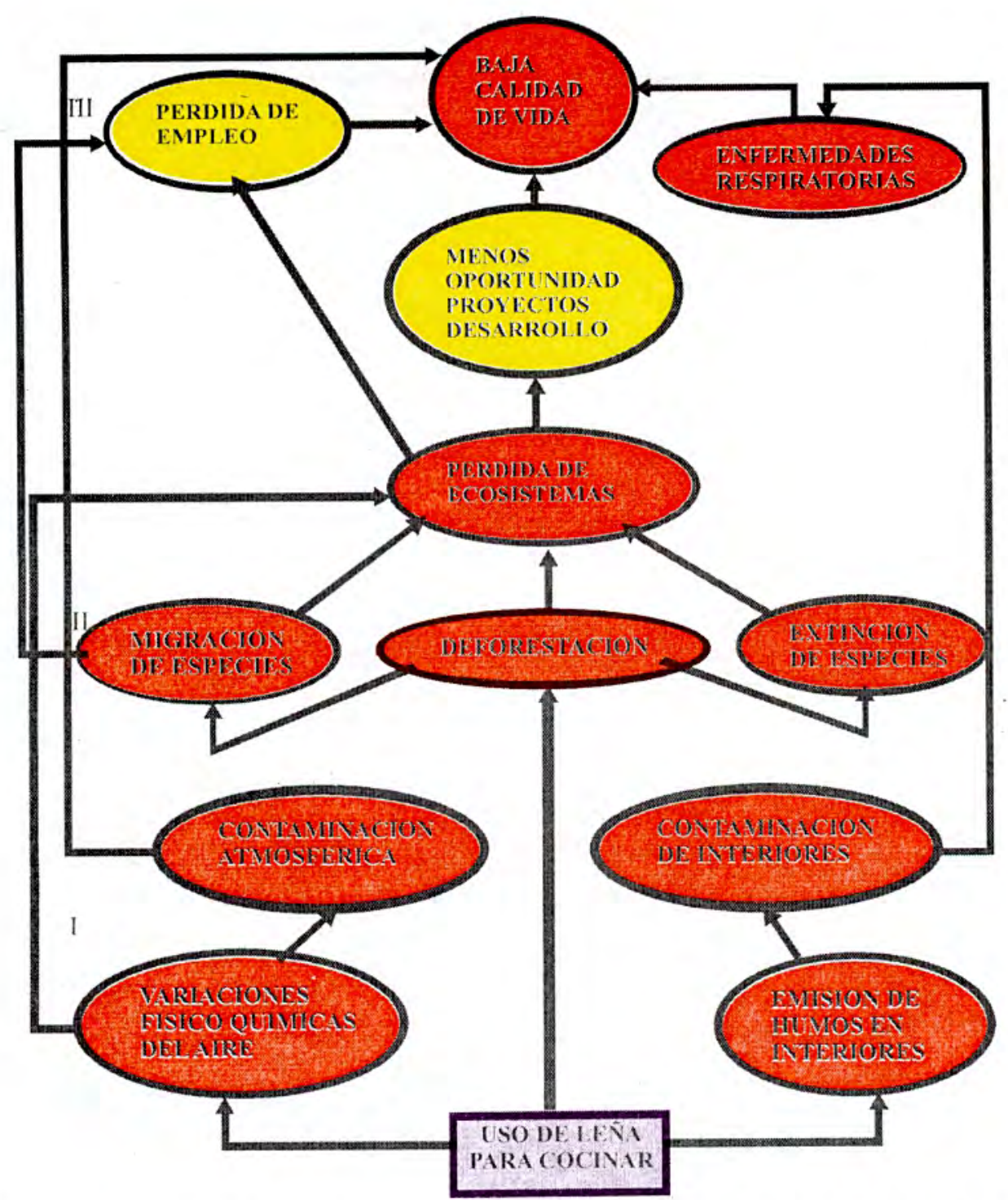

Figura 4. Diagrama de redes de impactos negativos debido a la extracción de leña de los bosques, matorrales y yaretales del ACRVM

estado inicial del componente ambiental; es decir, es beneficiosa o reduce la calidad ambiental. Es positivo (+) si mejora la calidad de un componente ambiental y es negativo (-) si reduce la calidad del mismo. En el diagrama de redes de la figura $4, \operatorname{los}$ números romanos indican lo siguiente: I Alteraciones en el Medio Físico: II Alteraciones en el Medio Biótico o Biológico y III Alteraciones en el medio Socio Económico y Cultural. Color rojo impacto altamente negativo, color amarillo moderadamente negativo.

\subsubsection{Evaluación Cuantitativa.}

Para la evaluación cuantitativa de impactos ambientales se ha aplicado la metodología de Leopold modificada de acuerdo a las caracteristicas de las actividades involucradas en el estudio de investigación. Se consideraron los siguientes criterios de evaluación: variación de la calidad ambiental ( $\left.\mathrm{V}_{\mathrm{c} .1}\right)$, magnitud $\left(\mathrm{M}_{\mathrm{g}}\right)$, extensión (Ex), persistencia (Per), capacidad de recuperación (Rec), sinergia (Si), significancia $(\mathrm{Sg})$. En esta parte se presenta la matriz de evaluación y valoración de impactos producidos por las actividades que conllevan a la obtención de leña para cocinar en el ACRVM de la Región Tacna. En la figura 5. Se presenta la matriz de valoración y cuantificación de impactos ambientales.

\subsubsection{Contaminación en interiores}

La combustión de la biomasa en fogones abiertos se da de manera incompleta e incontrolada y genera, por ello, una gran cantidad de particulas y gases contaminantes. De acuerdo con Cooper (1982, citado por Smith, 1987) la quema de biomasa en fogones abiertos genera diecisiete sustancias consideradas "contaminantes prioritarios" por la Agencia de Protección Ambiental de los Estados Unidos (EPA), para las cuales existe evidencia de toxicidad; más de catorce compuestos carcinógenos: seis tóxicos para los cilios y agentes mucocoagulantes; y cuatro precursores del cáncer.

En el interior de las viviendas, particularmente en las cocinas, ocurre la mayor exposición a estos contaminantes, siendo las mujeres y los niños las poblaciones mayormente expuestas por pasar la mayor 


\begin{tabular}{|c|c|c|c|c|c|c|c|c|c|c|}
\hline \multirow{2}{*}{\multicolumn{2}{|c|}{ Componente Ambiental }} & \multirow[b]{2}{*}{ Impacto } & \multicolumn{6}{|c|}{ Parámetro de valoración } & \multirow[b]{2}{*}{ 㘳 } & \multirow[b]{2}{*}{$\begin{array}{l}\text { Catificación de } \\
\text { Impacto }\end{array}$} \\
\hline & & & 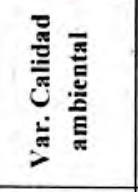 & 莺 & 产 & 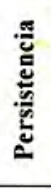 & 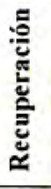 & 愛 & & \\
\hline \multirow{2}{*}{ 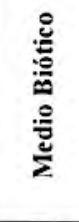 } & Flora & Deforestación & Negativo & 3 & 2 & 3 & 2 & 3 & -17 & $\begin{array}{l}\text { Altamente } \\
\text { Negativo }\end{array}$ \\
\hline & Fauna & $\begin{array}{l}\text { Migración y extinción de } \\
\text { especies }\end{array}$ & Negativo & 3 & 2 & 3 & 3 & 3 & -17 & $\begin{array}{l}\text { Altamente } \\
\text { Negativo }\end{array}$ \\
\hline \multirow{6}{*}{ 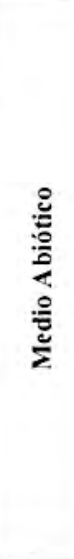 } & \multirow{4}{*}{ Calidad de aire } & $\begin{array}{c}\text { Contaminación del aire por } \\
\text { particulas en suspensión y gases } \\
\text { de combustión }\end{array}$ & Negativo & 3 & 1 & 2 & 1 & 3 & -13 & $\begin{array}{l}\text { Altamente } \\
\text { Negativo }\end{array}$ \\
\hline & & $\begin{array}{l}\text { Contaminación del aire por } \\
\text { compuestos volátiles }\end{array}$ & Negativo & 2 & 1 & 2 & 1 & 3 & -11 & $\begin{array}{l}\text { Moderadamente } \\
\text { Negativo }\end{array}$ \\
\hline & & Emisión de $\mathrm{CO}_{2}$ a la Atmóstera & Negativo & 1 & 1 & 2 & 1 & 0 & -6 & Baja Significancia \\
\hline & & Captura de $\mathrm{CO}_{2}$ & Negativo & 3 & 2 & 3 & 2 & 3 & -17 & $\begin{array}{l}\text { Altamente } \\
\text { Negativo }\end{array}$ \\
\hline & \multirow{2}{*}{$\begin{array}{l}\text { Calidad del } \\
\text { suelo }\end{array}$} & Paisaje & Negativo & 3 & 2 & 2 & 2 & 0 & -12 & $\begin{array}{l}\text { Moderadamente } \\
\text { Negativo } \\
\end{array}$ \\
\hline & & $\begin{array}{l}\text { Alteración de las caracteristicas } \\
\text { del suelo }\end{array}$ & Negativo & 3 & 1 & 3 & 2 & 3 & -15 & $\begin{array}{l}\text { Altamente } \\
\text { Negativo }\end{array}$ \\
\hline \multirow{6}{*}{ 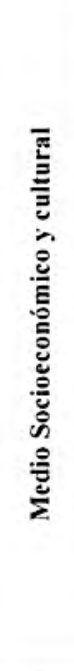 } & $\begin{array}{l}\text { Salud y } \\
\text { seguridad }\end{array}$ & $\begin{array}{c}\text { Riesgo de enfermedades y } \\
\text { quemaduras }\end{array}$ & Negativo & 2 & 1 & 2 & 2 & 3 & -12 & $\begin{array}{l}\text { Moderadamente } \\
\text { Negativo }\end{array}$ \\
\hline & Empleo & $\begin{array}{l}\text { Generación de empleo directo e } \\
\text { indirecto }\end{array}$ & Negativo & 2 & 2 & 2 & 2 & 0 & -10 & $\begin{array}{l}\text { Moderadamente } \\
\text { Negativo }\end{array}$ \\
\hline & $\begin{array}{l}\text { Alteración de } \\
\text { paisaje }\end{array}$ & $\begin{array}{c}\text { Alteración de la percepción } \\
\text { visual }\end{array}$ & Negativo $^{-}$ & 3 & 2 & 3 & 3 & 0 & -14 & $\begin{array}{l}\text { Altamente } \\
\text { Negativo }\end{array}$ \\
\hline & Migración & $\begin{array}{c}\text { Abandono de tierras y centros } \\
\text { poblados }\end{array}$ & Negativos & 2 & 2 & 2 & 2 & 0 & -10 & $\begin{array}{l}\text { Moderadamente } \\
\text { Negativo }\end{array}$ \\
\hline & $\begin{array}{l}\text { Conflictos } \\
\text { socio } \\
\text { ambientales }\end{array}$ & $\begin{array}{l}\text { Desequilibrio del medio por el } \\
\text { hombre }\end{array}$ & Negativo & 1 & 3 & 2 & 2 & 0 & -9 & $\begin{array}{l}\text { Moderadamente } \\
\text { Negativo }\end{array}$ \\
\hline & $\begin{array}{l}\text { Prestación de } \\
\text { servicios }\end{array}$ & $\begin{array}{l}\text { Pérdida de oportunidades para } \\
\text { brindar servicios ambientales }\end{array}$ & Negativo & 3 & 2 & 2 & 2 & 0 & -12 & $\begin{array}{l}\text { Moderadamente } \\
\text { Negativo }\end{array}$ \\
\hline
\end{tabular}

Figura 5. Matriz de Valoración y Calificación de Impactos Ambientales en el ACRVM de la Región Tacna

parte del tiempo en la cocina, están expuestas durante periodos de tres a seis horas diarios a niveles muy altos de contaminantes durante muchos años (Albalak, 1997). Las concentraciones de partículas superan ampliamente las concentraciones consideradas como permisibles de acuerdo con normas internacionales establecidas para la calidad del aire en ambientes exteriores.

\subsubsection{Impactos sobre la salud de los usuarios}

La exposición a contaminantes emitidos por fogones abiertos puede producir efectos adversos a la salud. La mayor cantidad de contaminantes se concentra en el interior de las viviendas y sus efectos a la salud dependen de factores como (Smith, 1987): Emisión, depende de la cantidad y tipo del combustible empleado $y$ caracteristicas de la combustión y estufa. Concentración, determinada por la cantidad de emisiones, por las condiciones interiores de ventilación y por la temperatura interior y exterior. Exposición, función directa del tiempo de permanencia en el ambiente contaminado $y$ de la concentración en el mismo. Dosis, depende del tiempo de exposición, de las condiciones de respiración y características del contaminante. Vulnerabilidad, la edad, sexo, estado socio-económico, historia clínica y estilo de vida son algunos factores determinantes que influyen en la vulnerabilidad de una persona. Existe evidencia consistente de que la contaminación intramuros debida a la utilización de biomasa al interior de la vivienda 
aumenta significativamente el riesgo de infecciones respiratorias agudas en niños y de enfermedades pulmonares obstructivas crónicas en adultos (WHO, 2002). Además hay evidencia de la relación entre la exposición a contaminantes y la incidencia de infecciones en las vías respiratorias superiores, otitis media, asma, cáncer de tracto nasofaríngeo y de la

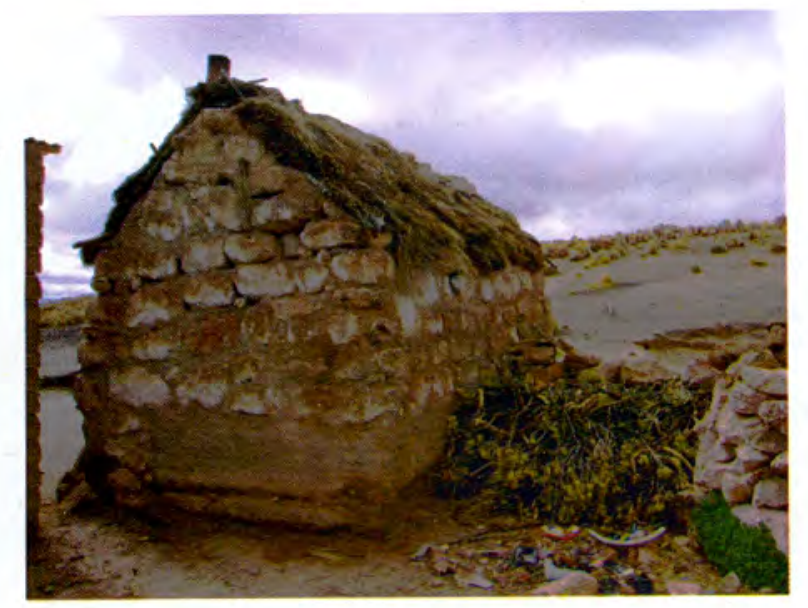

(a)

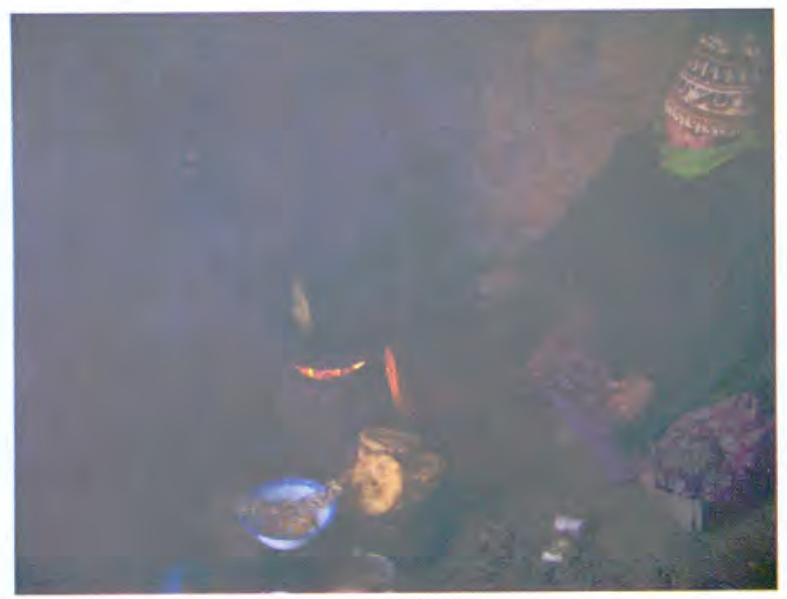

(c) laringe, tuberculosis pulmonar, insuficiencia ponderal del recién nacido y mortalidad de lactantes, así como de cataratas e infecciones oculares (Bruce et al., 2000). Una de las limitaciones principales de los estudios en salud humana es la falta de información sistemática y detallada de contaminación interior y de exposición personal.

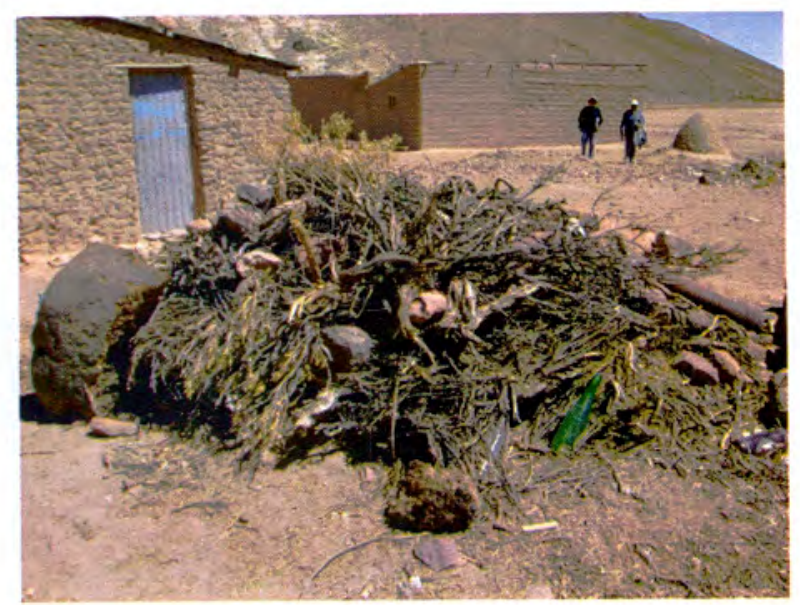

(b)

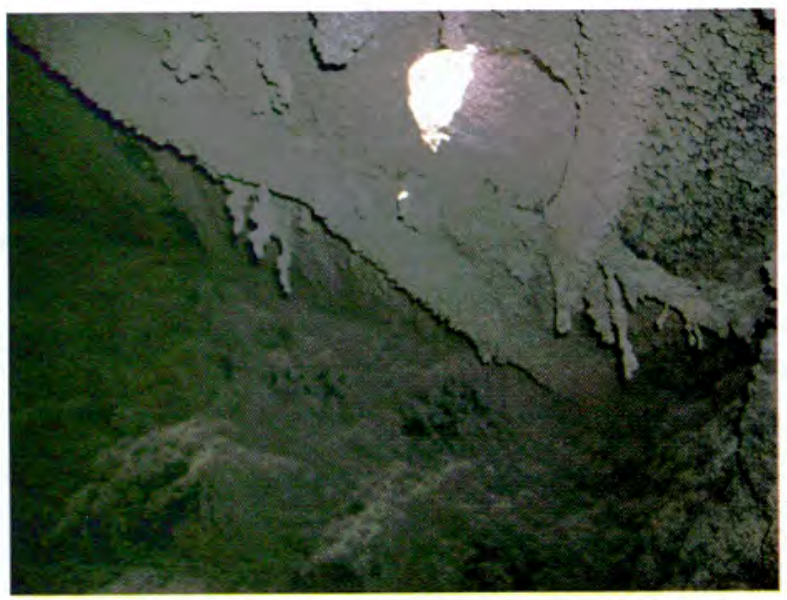

(d)

Figura 6. Fotos que muestran (a) las precarias viviendas de la gran mayoría de pobladores de las zonas rurales altoandinas, (b) la biomasa que se usa para cocinar alimentos, compuesta básicamente por queñoa, tola y yareta, (c) la contaminación intramuros en las viviendas rurales producida en las cocinas tradicionales y (d) el material particulado que se deposita en la parte interior de los techos de las viviendas rurales.

\section{CONCLUSIONES}

La demanda de leña en el Área de Conservación Regional Vilacota-Maure de la Región Tacna para la cocción de alimentos, es de magnitud considerable, lo cual permite concluir que la tasa de deforestación se mantiene en los valores tradicionales, a pesar que el recurso forestal ha disminuido por su explotación irracional pero ha sido reemplazado por otras especies sobre todo de matorrales como la Tola, y otras especies entre ellas la Yareta y también la bosta de camélidos sudamericanos.

Esta fuerte demanda de leña está causando una serie de impactos ambientales moderadamente negativos y altamente negativos, en los diferentes medios bióticos, abióticos, económicos y sociales en la población de la zona de estudio, que contribuye a incrementar los bajos niveles de calidad de vida de las comunidades que se encuentran dentro del ACRVM.

Los impactos ambientales más importantes son sobre los ecosistemas, flora, fauna lo cual está provocando que exista una creciente migración y extinción de especies, así como una paulatina pero constante degradación de los suelos por erosión causada por las lluvias y el sobre pastoreo principalmente.

El uso de leña para cocinar sus alimentos causa impactos negativos importantes en la población, sobre todo en aquellas personas que se encuentran la mayor parte del tiempo en la cocina, esto deberá ser verificado a través 
de un estudio epidemiológico detallado.

La introducción de cocinas mejoradas y cocinas solares parabólicas SK12, SK14 o SK98 pueden ser una alternativa de solución para solucionar la crisis de la leña que está ocurriendo en la zona en estudio, permitirá revertir la situación actual y además causará impacto positivos considerables, como la reducción de la deforestación, reducción de la migración y extinción de especies, reducción de enfermedades respiratorias, reducción de la degradación de los suelos, mejoras en la calidad de la dieta alimenticia y por lo tanto una mejor calidad de vida de la población.

\section{RECOMENDACIONES}

Se debe realizar un estudio de investigación epidemiológica de los impactos negativos directos sobre la salud de las personas, que trae como consecuencia el uso de la leña para la cocción de alimentos en cocinas tradicionales en el ACRVM, principalmente sobre las vias respiratorias y el sentido de la vista.

Es necesario realizar un estudio detallado de los impactos que sobre las especies de flora y fauna tiene la extracción de la leña, particularmente de la Queñoa, el Suri. la Tola, la Yareta y otras especies que son vulnerables, asi como en el suelo y el recurso hídrico subterráneo.

Es necesario que las municipalidades y los gobiernos regionales consideren en sus planes estratégicos de desarrollo la implementación de un programa de capacitación y difusión para el uso masivo de las cocinas mejoradas y solares en el Área de Conservación Regional Vilacota-Maure de Tacna.

\section{REFERENCIAS BIBLIOGRÁFICAS}

CEPIS-OPS/OMS, Evaluación de Impacto Ambiental. http//www.cepis.ops-oms.org

Conesa Fdez, V., (1997), Guia Metodológica para la Evaluación de Impacto Ambiental, 3ra edición, Ediciones Mundi - Prensa, Madrid, España

CAMDESO-GRT. (2007), Plan Maestro del ACRVMRegión Tacna, Tacna, Perú.

Franco Diaz, J.M.. (2007), Diagnóstico Socioeconómico del Área de Conservación Regional Vilacota Maure, GRT - Gerencia Regional del Medio Ambiente y Recursos Naturales, Tacna, Perú.

GIRA, (2004), El uso de estufas mejoradas de leña en los hogares: Evaluación de Reducciones en la Exposición Personal, Informe Final, Morelia, México. http://www.gira.org.mx

GIRA, (2003), El uso de biomasa como fuente de energía en los hogares: efectos en el ambiente y la salud. y posibles soluciones, Informe Final, Morelia, México. http://www.gira.org.mx

GTZ, CEPIS, OPS/OMS MINSA, (2005) La Implementación de Cocinas Saludables como intervención clave en el mejoramiento de la Salud Ambiental en los Andes. Lima. Perú.

IDMA, Programa de Desarrollo Sostenible (1998). "Estudio de Demanda de Leña y su Impacto Ambiental en el Santuario Nacional de Ampay", Abancay, Perú

Lampinen, Ari, (1994), "La Reducción de Deforestación Tropical por el uso masivo de Fogones solares", Technology for Life, Finlandia

O Ryan Curtis, D. (2006), Solar Cookyng and Health. ITDG-Revista Boiling Point $\mathrm{N}^{\circ} 52$, pp 30-32.

http:/www.itdg.org/?id=boiling point

OPS/OMS-MINSA.(2006), Seminario Polución del Aire Domestico (PAD) por Fogones Tradicionales en el Perú: Sistematización. Lima. Perú.

Polo Bravo, C. y Torres Muro H.. (2002), Energía y Desarrollo Sostenible, Centro de Energias Renovables de Tacna-UNJBG, Memorias impresas del IX SPES, APES-UNSA, Arequipa. Perú

PNUD, BUN-CA, GEF, (2002), Manuales sobre Energia Renovables: Biomasa, I ra Edición, San José, Costa Rica

Seifer D. y Vilchez M., (1999), La Cocina Solar SK98M, Benicarló

Seifert Dieter, (2000), Pasos a un Programa Global de la Cocina Solar, Encuentro Solar 2000, Benicarló

Seifert Dieter, (1998). Propuesta para la Financiación de un Proyecto Global de Cocinas Solares mediante Joint Implementation, $11^{\circ}$ Foro Solar Internacional, Köln, Alemania

Torres Muro, H., Polo Bravo C. y López Cornejo O., (2003). Impacto de las Cocinas Solares sobre la Demanda de Leña y la Emisión de Dióxido de Carbono en la Zona Altoandina de Tacna". CERT-UNJBG, Informe Final de Proyecto de Investigación, COINUNJBG. Tacna, Perú

Torres Muro, H., Polo Bravo C., (2004), Perspectiva Ambiental de las Cocinas Solares en la Zona Altoandina de Tacna, Revista "CIENCIA Y DESARROLLO, $\mathrm{N}^{\circ} 08$, Consejo de Investigación-UNJBG, Tacna, Perú

Correspondencia:

Hugo Alfredo Torres Muro

Centro de Energias Renovables de Tacna

Av. Miraflores s/n. Tacna. Perú

hugotorres@hotmail.com 